Article

\title{
Inhomogeneous phases in the chirally imbalanced $2+1$-dimensional Gross-Neveu model and their absence in the continuum limit
}

\author{
Laurin Pannullo $^{1 *}$ (D), Marc Wagner 1,2 (D) and Marc Winstel ${ }^{1 *}$ (D) \\ 1 Institut für Theoretische Physik, Goethe Universität Frankfurt am Main, 60438 Frankfurt am Main, \\ Germany \\ 2 Helmholtz Research Academy Hesse for FAIR, Campus Riedberg, Max-von-Laue-Straße 12, 60438 \\ Frankfurt am Main, Germany \\ * Correspondence: pannullo@itp.uni-frankfurt.de (L.P.), winstel@itp.uni-frankfurt.de (M.Wi.)
}

\begin{abstract}
We study the $\mu-\mu_{45}-T$ phase diagram of the $2+1$-dimensional Gross-Neveu model, where $\mu$ denotes the ordinary chemical potential, $\mu_{45}$ the chiral chemical potential and $T$ the temperature. We use the mean-field approximation and two different lattice regularizations with naive chiral fermions. An inhomogeneous phase at finite lattice spacing is found for one of the two regularizations. Our results suggest that there is no inhomogeneous phase in the continuum limit. We show that a chiral chemical potential is equivalent to an isospin chemical potential. Thus, all results presented in this work can also be interpreted in the context of isospin imbalance.
\end{abstract}

Keywords: inhomogeneous phases, chiral imbalance, isospin imbalance, $2+1$ dimensional field theories, Gross-Neveu model, mean-field

\section{Introduction}

The Gross-Neveu (GN) model describes a theory of $N_{f}$ fermion flavors with a quartic interaction. It is a rather simple model commonly used to explore and to describe the spontaneous breaking of chiral symmetry [1] in the $\mu$-T plane, where $\mu$ denotes the chemical potential and $T$ the temperature. In the limit $N_{f} \rightarrow \infty$ (corresponding to the mean-field approximation or, equivalently, the neglect of bosonic quantum fluctuations) the 1+1-dimensional GN model exhibits three phases: a symmetric phase (with a vanishing chiral condensate), a homogeneous symmetry-broken phase (with a nonzero, but spatially constant condensate) and an inhomogeneous phase, where the chiral condensate is an oscillating function of space [2-4]. The phase diagrams of the GN model and related theories were also investigated at finite $N_{f}$, i.e., with bosonic quantum fluctuations included, using lattice Monte-Carlo simulations [5-10] and the functional renormalization group (FRG) [11].

Inhomogeneous phases are not limited to the GN model, but were found in several models in the mean-field approximation in $1+1$ dimensions [12-15] and in $3+1$ dimensions [16-19]. For a review we refer to Ref. [20]. In recent works [21-24] it has been discussed that inhomogeneous phases might be related to so-called moat regimes, where the bosonic wave function renormalization $Z$ is negative. Such a regime has been found in an FRG study of the phase diagram of quantum chromodynamics (QCD) [25]. A similar regime has been observed in the 1+1-dimensional GN model (see Fig. 9 of Ref. [26], where the wave function renormalization $Z$ is plotted in the $\mu-T$ plane). One finds that a negative $Z$ accompanies the instability of a homogeneous condensate with respect to inhomogeneous perturbations as a necessary, but not a sufficient condition. The possible existence of a moat regime in the QCD phase diagram encourages to improve our understanding of inhomogeneous condensation and related phenomena in QCD-inspired models. 
Recently, the existence of inhomogeneous phases was also explored in the $2+1$ dimensional GN model in the mean-field approximation [27-29]. Such 2+1-dimensional four-fermion theories are of interest both in high energy physics [30-34] and in condensed matter physics [35-42], but also to study conceptual questions, e.g., renormalizability in the $1 / N$ expansion or in a perturbative approach [43-46]. Hence, confirming the existence of an inhomogeneous phase in such a model could have a significant impact. Early seminal studies of the $\mu$-T phase diagram of the $2+1$-dimensional GN model $[47,48]$ have reported a second order phase transition between the symmetric and the homogeneous symmetry-broken phase at finite $T$ and $\mu$ and a first order phase transition at $T=0$. However, in these studies only a homogeneous order parameter was considered. In our recent publication Ref. [29] we have studied the existence of an inhomogeneous phase in the $2+1$-dimensional GN model within the mean-field approximation. Our main findings are that an inhomogeneous phase is present at finite regulator and for certain regularization schemes (a Pauli-Villars cutoff and a specific lattice discretization), but it disappears, when the regulator is removed, as it was previously observed in Ref. [34].

In this work we continue our investigations from Ref. [29] by extending the $2+1$ dimensional GN model with a chiral chemical potential. We study its phase diagram, where our main focus is on possibly existing inhomogeneous phases. While the GN model might be too simple to realistically describe the effect of chiral imbalance on QCD, it might still improve our conceptual understanding of inhomogeneous condensation in the presence of chiral imbalance, which is an important problem. A difference in the densities of left and right handed quarks is relevant in physical systems such as heavy-ion collisions $[49,50]$ or compact stars $[51,52]$. The impact of chiral imbalance on chiral symmetry breaking has been studied, e.g., in $1+1$-dimensional models [13], where it had no influence on the existence of the inhomogeneous chiral spiral, and in 3 + 1-dimensional models [53-55], where only homogeneous order parameters were considered. A chirally imbalanced $2+1$-dimensional GN model, extended by a quartic difermion interaction, was explored in Refs. [56,57] with the aim to clarify the competition of homogeneous fermion-fermion condensation and homogeneous chiral condensation. In recent two-color and three-color QCD studies [58,59] a chiral chemical potential has been investigated, which has been found to increase the chiral transition temperature. This result is supported by a Nambu-Jona-Lasinio (NJL) model study [60].

This paper is structured as follows. We start in Section 2 by discussing the theoretical basics of the GN model in $2+1$ dimensions including details on the underlying chiral symmetry. We also add a chiral chemical potential to the model and show the equivalence of chiral imbalance and isospin imbalance. In Section 3 we discretize the effective action of the model using lattice field theory. Section 4 is the main part of our paper, where numerical results are presented and discussed. Finally, we conclude in Section 5. Preliminary results from this project were presented at a recent conference [61].

\section{Theoretical basics}

\subsection{Action and partition function}

The action of the GN model in $2+1$ dimensions with $N_{f}$ fermion flavors is

$$
S[\bar{\psi}, \psi]=\int d^{3} x\left(\sum_{n=1}^{N_{f}} \bar{\psi}_{n}\left(\gamma_{\nu} \partial_{\nu}+\gamma_{0} \mu\right) \psi_{n}-\frac{g^{2}}{2}\left(\sum_{n=1}^{N_{f}} \bar{\psi}_{n} \psi_{n}\right)^{2}\right)
$$

where $\psi_{n}$ represent $N_{f}$ massless fermion fields, $\mu$ is the chemical potential and $g^{2}$ is the coupling of the four-fermion interaction. $\int d^{3} x=\int_{0}^{1 / T} d x_{0} \int_{\mathbb{R}^{2}} d^{2} x$ with $d^{2} x=d x_{1} d x_{2}$ and $T$ denoting the temperature given by the inverse extent of the periodic temporal direction of Euclidean space-time. 
The action (2.1) is equivalent to

$$
S[\bar{\psi}, \psi, \sigma]=\int d^{3} x\left(\frac{N_{f}}{2 \lambda} \sigma^{2}+\sum_{n=1}^{N_{f}} \bar{\psi}_{n} Q[\mu, \sigma] \psi_{n}\right),
$$

where $\sigma$ is a scalar boson field, $\lambda=N_{f} g^{2}$ is the rescaled coupling and

$$
Q[\mu, \sigma]=\gamma_{v} \partial_{v}+\gamma_{0} \mu+\sigma
$$

is the Dirac operator. Integration over the fermion fields leads to the so-called effective action and the corresponding partition function

$$
S_{\text {eff }}[\sigma]=N_{f}\left(\frac{1}{2 \lambda} \int d^{3} x \sigma^{2}-\ln \operatorname{Det} Q[\mu, \sigma]\right), \quad Z=\int D \sigma e^{-S_{\text {eff }}[\sigma]} .
$$

One can show that $\langle\sigma(x)\rangle$ is related to the condensate $\left\langle\bar{\psi}_{n}(x) \psi_{n}(x)\right\rangle$ according to

$$
\langle\sigma(x)\rangle=-\frac{\lambda}{N_{f}}\left\langle\bar{\psi}_{n}(x) \psi_{n}(x)\right\rangle .
$$

In this work we restrict the dependence of $\sigma$ to the spatial coordinates, i.e., $\sigma=\sigma(\mathbf{x})$. With this restriction $S_{\text {eff }}[\sigma]$ is real, which is shown in Appendix A.2 of Ref. [29].

Since $S_{\text {eff }}[\sigma] \propto N_{f}$, the limit $N_{f} \rightarrow \infty$ reduces the relevant configurations in the partition function (2.4) to the global minima of $S_{\text {eff }}[\sigma]$. Thus, the computation of a path integral is reduced to an optimization problem. In the case of degenerate global minima, spontaneous symmetry breaking selects one of these minima. Consequently, an expectation value $\langle O(\sigma)\rangle$ is identical to the value of $O$ evaluated at the corresponding global minimum, i.e., $\langle O(\sigma)\rangle \rightarrow O(\sigma)$. In particular, $\langle\sigma\rangle \rightarrow \sigma$. For the remainder of this paper we consider exclusively the limit $N_{f} \rightarrow \infty$.

\subsection{Representation of the Dirac matrices and chiral symmetry}

Typically one uses either an irreducible $2 \times 2$ representation or a reducible $4 \times 4$ representation of the Dirac algebra for the $\gamma$ matrices appearing in the Dirac operator (2.3) (for details see, e.g., Refs. [29,32,33,62]. In case of an irreducible $2 \times 2$ representation there is no symmetry, which can be interpreted as chiral symmetry, because it is impossible to define a matrix $\gamma_{5}$, which anticommutes with $\gamma_{0}, \gamma_{1}$ and $\gamma_{2}$. Therefore, a reducible $4 \times 4$ representation is more appropriate in our context, e.g.,

$$
\begin{aligned}
& \gamma_{0}=\tau_{3} \otimes \tau_{2}=\left(\begin{array}{cc}
+\tau_{2} & 0 \\
0 & -\tau_{2}
\end{array}\right), \quad \gamma_{1}=\tau_{3} \otimes \tau_{3}=\left(\begin{array}{cc}
+\tau_{3} & 0 \\
0 & -\tau_{3}
\end{array}\right), \\
& \gamma_{2}=\tau_{3} \otimes \tau_{1}=\left(\begin{array}{cc}
+\tau_{1} & 0 \\
0 & -\tau_{1}
\end{array}\right),
\end{aligned}
$$

where $\tau_{j}$ denote the Pauli matrices. The three matrices $+\tau_{1},+\tau_{2}$ and $+\tau_{3}$ as well as the three matrices $-\tau_{1},-\tau_{2}$ and $-\tau_{3}$ form irreducible $2 \times 2$ representations, which are inequivalent. The corresponding upper two and lower two entries of the fermion fields $\psi_{n}$ can be interpreted as left-handed and right-handed components, respectively.

The Dirac operator (2.3) is then block-diagonal,

$$
Q[\mu, \sigma]=Q^{(4)}[\mu, \sigma]=\left(\begin{array}{cc}
Q^{(2)}[\mu, \sigma] & 0 \\
0 & \tilde{Q}^{(2)}[\mu, \sigma]
\end{array}\right),
$$


where

$$
\begin{aligned}
& Q^{(2)}[\mu, \sigma]=+\tau_{2}\left(\partial_{0}+\mu\right)+\tau_{3} \partial_{1}+\tau_{2} \partial_{2}+\sigma \\
& \tilde{Q}^{(2)}[\mu, \sigma]=-\tau_{2}\left(\partial_{0}+\mu\right)-\tau_{3} \partial_{1}-\tau_{2} \partial_{2}+\sigma
\end{aligned}
$$

represent Dirac operators for left-handed and right-handed fermion fields $\psi_{n}^{L / R}$ (see also Eq. (14) and Eq. (15) in Ref. [29]). One can show that $\operatorname{Det} Q^{(2)}[\mu, \sigma]$ and $\operatorname{Det} \tilde{Q}^{(2)}[\mu, \sigma]$ are invariant under both $\mu \rightarrow-\mu$ and $\sigma \rightarrow-\sigma$. Using the latter one can show

$$
\operatorname{Det} Q^{(2)}[\mu, \sigma]=\operatorname{Det} \tilde{Q}^{(2)}[\mu, \sigma] \text {. }
$$
tions ${ }^{1}$

The action with $Q=Q^{(4)}[\mu, \sigma]$ is invariant under the discrete chiral transforma-

$$
\begin{array}{ll}
\psi_{n} \rightarrow \gamma_{4} \psi_{n}, & \bar{\psi}_{n} \rightarrow-\bar{\psi}_{n} \gamma_{4} \\
\psi_{n} \rightarrow \gamma_{5} \psi_{n}, & \bar{\psi}_{n} \rightarrow-\bar{\psi}_{n} \gamma_{5}
\end{array}
$$

with

$$
\gamma_{4}=\tau_{1} \otimes \mathbb{1}_{2}=\left(\begin{array}{cc}
0 & +\mathbb{1}_{2} \\
+\mathbb{1}_{2} & 0
\end{array}\right), \quad \gamma_{5}=-\tau_{2} \otimes \mathbb{1}_{2}=\left(\begin{array}{cc}
0 & +\mathrm{i} \mathbb{1}_{2} \\
-\mathrm{i} \mathbb{1}_{2} & 0
\end{array}\right) .
$$

Both $\gamma_{4}$ and $\gamma_{5}$ anticommute with $\gamma_{0}, \gamma_{1}$ and $\gamma_{2}$ and, thus, fulfill the necessary properties for generating an axial chiral transformation. The symmetries (2.11) and (2.12) are also present for the action (2.2), where the corresponding transformation of $\sigma$ is in both cases $\sigma \rightarrow-\sigma$. Thus, $\sigma$ is an order parameter for chiral symmetry breaking ${ }^{2}$.

In addition to the transformations (2.11) and (2.12) the action is also invariant under the continuous vector chiral transformations

$$
\begin{aligned}
& \psi_{n} \rightarrow \mathrm{e}^{\mathrm{i} \alpha^{a} T^{a}} \psi_{n}, \quad \bar{\psi}_{n} \rightarrow \bar{\psi}_{n} \mathrm{e}^{-\mathrm{i} \alpha^{a} T^{a},}, \\
& \psi_{n} \rightarrow \mathrm{e}^{\mathrm{i} \beta^{a} T^{a} \gamma_{45}} \psi_{n}, \quad \bar{\psi}_{n} \rightarrow \bar{\psi}_{n} \mathrm{e}^{-\mathrm{i} \beta^{a} T^{a} \gamma_{45},}
\end{aligned}
$$

where $T^{a}$ denote the generators of $\mathrm{U}\left(N_{f}\right)$ flavor rotations and

$$
\gamma_{45}=\mathrm{i} \gamma_{4} \gamma_{5}=\tau_{3} \otimes \mathbb{1}_{2}=\left(\begin{array}{cc}
+\mathbb{1}_{2} & 0 \\
0 & -\mathbb{1}_{2}
\end{array}\right)
$$

(see also Refs. [32,62]).

The transformations (2.11), (2.12) and (2.15) are not independent. For example, ( 2.11) can be written as combination of (2.12) and (2.15) with $\beta^{a} T^{a}=-\pi / 2$,

$$
\psi_{n} \rightarrow \mathrm{e}^{\mathrm{i}(-\pi / 2) \gamma_{45}} \gamma_{5} \psi_{n}=\gamma_{4} \psi_{n}, \quad \bar{\psi}_{n} \rightarrow-\bar{\psi}_{n} \gamma_{5} \mathrm{e}^{-\mathrm{i}(-\pi / 2) \gamma_{45}}=-\bar{\psi}_{n} \gamma_{4} .
$$

Thus, there is only one independent $\mathbb{Z}_{2}$ symmetry, i.e., the structure of chiral symmetry is $\mathrm{U}_{\mathbb{1}}\left(N_{f}\right) \times \mathrm{U}_{\gamma_{45}}\left(N_{f}\right) \times \mathbb{Z}_{2}$.

1 For free fermions one can define continuous axial chiral symmetry transformations with both $\gamma_{4}$ and $\gamma_{5}$. The four-fermion interaction in Eq. (2.1) breaks the corresponding symmetries explicitely (see, e.g., Ref. [29]).

2 When using an irreducible $2 \times 2$ fermion representation, there is no chiral symmetry. $\sigma$, however, can still be interpreted as order parameter for parity breaking. 
A chiral chemical potential $\mu_{45}$ can be introduced in a straightforward way by extending and replacing the Dirac operator in (2.3) or equivalently (2.7) according to

$$
\begin{gathered}
Q[\mu, \sigma]=Q^{(4)}[\mu, \sigma] \rightarrow Q\left[\mu, \mu_{45}, \sigma\right]=Q^{(4)}\left[\mu, \mu_{45}, \sigma\right]=\gamma_{v} \partial_{v}+\gamma_{0} \mu+\gamma_{45} \gamma_{0} \mu_{45}+\sigma= \\
=\left(\begin{array}{cc}
Q^{(2)}\left[\mu+\mu_{45}, \sigma\right] & 0 \\
0 & \tilde{Q}^{(2)}\left[\mu-\mu_{45}, \sigma\right]
\end{array}\right) .
\end{gathered}
$$

$\mu_{45}$ contributes to the chemical potentials of the left-handed (upper two) components and the right-handed (lower two) components with opposite sign and, thus, causes chiral imbalance. We note that there are other possibilities to define chirality and chiral imbalance (see, e.g., Refs. [56,57] and Section 5) differing from our definition, where leftand right-handed fermion fields $\psi_{n}^{L / R}$ are projected from the fermion fields as

$$
\psi_{n}^{L / R}=P^{L / R} \psi_{n}=\frac{1}{2}\left(\mathbb{1}_{4} \pm \gamma_{45}\right) \psi_{n}
$$

with $P^{L / R}$ denoting the corresponding projectors.

As done for $\mu_{45}=0$ in appendix A of Ref. [29], one can show that $\operatorname{Det} Q^{(4)}\left[\mu, \mu_{45}, \sigma\right]$ is invariant under both $\left(\mu, \mu_{45}\right) \rightarrow\left(-\mu,-\mu_{45}\right)$ and $\sigma \rightarrow-\sigma$. Since

$$
\operatorname{Det} Q^{(4)}\left[\mu, \mu_{45}, \sigma\right]=\operatorname{Det} Q^{(2)}\left[\mu+\mu_{45}, \sigma\right] \operatorname{Det} \tilde{Q}^{(2)}\left[\mu-\mu_{45}, \sigma\right]
$$

Det $Q^{(4)}\left[\mu, \mu_{45}, \sigma\right]$ is also invariant under the exchange of the ordinary and the chiral chemical potential, $\mu \leftrightarrow \mu_{45}$. Clearly, $S_{\text {eff }}[\sigma]$ as well as the phase diagram share this invariance. In Section 4 we use this property to cross-check our numerical results.

We note that the effective action can be written as sum of a left-handed and a right-handed part,

$$
\begin{aligned}
S_{\mathrm{eff}}[\sigma] & =N_{f}\left(\frac{1}{2 \lambda} \int d^{3} x \sigma^{2}-\ln \operatorname{Det} Q^{(4)}\left[\mu, \mu_{45}, \sigma\right]\right)=S_{\mathrm{eff}}^{L}[\sigma]+S_{\mathrm{eff}}^{R}[\sigma]= \\
& =\sum_{X=L, R} \underbrace{N_{f}\left(\frac{1}{2(2 \lambda)} \int d^{3} x \sigma^{2}-\ln \operatorname{Det} Q^{(2)}\left[\mu_{X}, \sigma\right]\right)}_{=S_{\mathrm{eff}}^{X}[\sigma]}
\end{aligned}
$$

with $\mu_{L}=\mu+\mu_{45}$ and $\mu_{L}=\mu-\mu_{45}$. Of course, the two parts are not independent, but coupled via $\sigma$. Moreover, both parts are equivalent to the chirally balanced effective action (see Section 2.3 of Ref. [29]), i.e.

$$
S_{\text {eff }}^{L}[\sigma]=\left.\frac{1}{2} S_{\text {eff }}[\sigma]\right|_{\mu=\mu_{L}, \mu_{45}=0}, \quad S_{\text {eff }}^{R}[\sigma]=\left.\frac{1}{2} S_{\text {eff }}[\sigma]\right|_{\mu=\mu_{R}, \mu_{45}=0} .
$$

This property will be useful, when we discuss our numerical results in Section 4.

\subsection{Equivalence of isospin and chiral imbalance}

In this subsection we consider an even number of fermion flavors $N_{f}$, again in the 4-component reducible representation, and assign half of them (the " $u$ flavors") a chemical potential $\mu+\mu_{\mathrm{I}}$, the other half (the "d flavors") a chemical potential $\mu-\mu_{\mathrm{I}}$. Clearly, $\mu_{\mathrm{I}}$ generates an imbalance between the $u$ and the $d$ flavors and, thus, can be interpreted as isospin chemical potential.

The corresponding effective action is

$$
S_{\mathrm{eff}, \mathrm{I}}[\sigma]=N_{f}\left(\frac{1}{2 \lambda} \int d^{3} x \sigma^{2}-\frac{1}{2} \ln \operatorname{Det} Q_{\mathrm{I}}\left[\mu, \mu_{I}, \sigma\right]\right),
$$


where the Dirac operator is an $8 \times 8$ matrix in spin and isospin space,

$$
\begin{aligned}
Q_{\mathrm{I}}\left[\mu, \mu_{I}, \sigma\right] & =\gamma_{v} \partial_{v}+\gamma_{0} \mu+\gamma_{0} \tau_{3} \mu_{\mathrm{I}}+\sigma= \\
& =\left(\begin{array}{cc}
Q^{(4)}\left[\mu+\mu_{\mathrm{I}}, 0, \sigma\right] & 0 \\
0 & Q^{(4)}\left[\mu-\mu_{\mathrm{I}}, 0, \sigma\right]
\end{array}\right) .
\end{aligned}
$$

Using Eq. (2.20) one can show

$$
\ln \operatorname{Det} Q_{\mathrm{I}}\left[\mu, \mu_{I}, \sigma\right]=2 \ln \operatorname{Det} Q^{(4)}\left[\mu, \mu_{\mathrm{I}}, \sigma\right] .
$$

Consequently, the effective action for the GN model with isospin imbalance, Eq. (2.23), is identical to the the effective action for the GN model with chiral imbalance, Eq. (2.21), when identifying $\mu_{\mathrm{I}}=\mu_{45}$. Thus, all numerical results presented in Section 4 can either be interpreted in the context of chiral imbalance or of isospin imbalance. We note that this equivalence of isospin and chiral imbalance is specific to the GN model in $2+1$ dimensions.

\section{Lattice discretization}

We use a lattice discretization of the effective action (2.21), which is similar to the discretization discussed in Section 4 of our previous work [29]. The key difference is that we use the naive fermion discretization also in temporal and not only in the spatial directions.

We consider a 3-dimensional space-time volume $\beta V$, where $\beta=1 / T$ is the inverse temperature and $V=L^{2}$ the quadratic spatial volume. The boundary conditions are periodic in the 2 spatial directions and periodic and antiperiodic in temporal direction for the fields $\sigma$ and $\psi_{n}, \bar{\psi}_{n}$, respectively. We use a cubic lattice with $N_{t} \times N_{s}^{2}$ lattice sites and lattice spacing $a$, i.e., $\beta=a N_{t}$ and $L=a N_{s}$. In the following all dimensionful quantities are expressed in units of the lattice spacing, e.g. $a \equiv 1, \beta \equiv \beta / a$, etc. Because of the finite space-time volume, the 3 -dimensional momenta are quantized,

$$
p=\left(p_{0}, \mathbf{p}\right)=2 \pi\left(\frac{k_{0}+\eta}{N_{t}}, \frac{\mathbf{k}}{N_{s}}\right)
$$

with

$$
k_{0} \in\left\{-\frac{N_{t}}{2},-\frac{N_{t}}{2}+1, \ldots, \frac{N_{t}}{2}-1\right\} \quad \text { and } \quad \mathbf{k}_{i} \in\left\{-\frac{N_{s}}{2},-\frac{N_{s}}{2}+1, \ldots, \frac{N_{s}}{2}-1\right\}
$$

and $\eta=0,1 / 2$ corresponding to periodic and antiperiodic boundary conditions in temporal direction.

In our numerical implementation the effective action and the fields are treated in momentum space,

$$
\frac{S_{\text {eff }}[\sigma]}{N_{f}}=\frac{N_{t} N_{s}^{2}}{2 \lambda} \sum_{\mathbf{p}} \tilde{\sigma}^{2}(\mathbf{p})-\frac{1}{8} \ln \operatorname{Det} \tilde{Q}^{(4)}\left[\mu, \mu_{45}, \sigma\right],
$$

where

$$
\tilde{\sigma}(\mathbf{p})=\frac{1}{N_{S}^{2}} \sum_{\mathbf{x}} \sigma(\mathbf{x}) \mathrm{e}^{\mathrm{ix} \cdot \mathbf{p}}
$$


are the Fourier coefficients of the field $\sigma(\mathbf{x})$.

$$
\begin{gathered}
\tilde{Q}_{p, q}^{(4)}\left[\mu, \mu_{45}, \sigma\right]=N_{t} N_{s}^{2}\left(\mathrm{i} \delta_{p, q} \sum_{v=0}^{2} \gamma_{v} \sin \left(p_{v}-\delta_{v, 0} \mathrm{i}\left(\mu+\gamma_{45} \mu_{45}\right)\right)+\right. \\
\left.+\delta_{p_{0}, q_{0}} \tilde{W}_{2}(\mathbf{p}-\mathbf{q}) \tilde{\sigma}(\mathbf{p}-\mathbf{q})\right)
\end{gathered}
$$

is the Dirac operator in momentum space. On the lattice this operator is a matrix with columns and rows labeled by the momenta $p$ and $q$, respectively. The sin for $v=0$ contains the matrix $\gamma_{45}$, but can be simplified according to

$$
\sin \left(p_{0}-\mathrm{i}\left(\mu+\gamma_{45} \mu_{45}\right)\right)=\sin \left(p_{0}-\mathrm{i} \mu\right) \cos \left(\mathrm{i} \mu_{45}\right)-\gamma_{45} \cos \left(p_{0}-\mathrm{i} \mu\right) \sin \left(\mathrm{i} \mu_{45}\right) .
$$

An appropriately chosen weight function $\tilde{W}_{2}(\mathbf{p})$ is necessary to ensure the correct continuum limit (see Refs. $[5,7,29]$ for details). We investigate and compare two possible choices,

$$
\begin{aligned}
& \tilde{W}_{2}(\mathbf{p})=\tilde{W}_{2}^{\prime}(\mathbf{p})=\prod_{v=1,2} \tilde{W}_{1}^{\prime}\left(p_{v}\right), \quad \tilde{W}_{1}^{\prime}\left(p_{v}\right)=\frac{\cos \left(p_{v}\right)+1}{2} \\
& \tilde{W}_{2}(\mathbf{p})=\tilde{W}_{2}^{\prime \prime}(\mathbf{p})=\prod_{v=1,2} \tilde{W}_{1}^{\prime \prime}\left(p_{v}\right), \quad \tilde{W}_{1}^{\prime \prime}\left(p_{v}\right)=\Theta\left(\pi / 2-\left|p_{v}\right|\right)
\end{aligned}
$$

with $\Theta$ denoting the Heaviside function.

Because of the restriction of $\sigma$ to the spatial coordinates, i.e. $\sigma=\sigma(\mathbf{x})$, the Dirac operator (3.4) is block-diagonal with respect to $p_{0}$ and $q_{0}$. This simplifies the computation of Det $\tilde{Q}^{(4)}\left[\mu, \mu_{45}, \sigma\right]$ to the computation of $N_{t}$ determinants of smaller matrices of size $4 N_{s}^{2} \times 4 N_{s}^{2}$.

\section{Numerical results}

Using lattice field theory the phase diagram of the $2+1$-dimensional GN model with $\mu_{45}=0$ was extensively explored in Refs. [27-29]. There is a symmetric phase with $\sigma=0$ at large $\mu$ or large $T$ and a homogeneous symmetry-broken phase with a constant $\sigma=\bar{\sigma}$ at small $\mu$ and small $T$. Moreover, at finite lattice spacing and for certain discretizations (e.g., $\tilde{W}_{2}=\tilde{W}_{2}^{\prime \prime}$ ) there is additionally an inhomogeneous phase, where $\sigma(\mathbf{x})$ is a varying function of the spatial coordinates. However, this inhomogeneous phase shrinks, when decreasing the lattice spacing, and seems to vanish in the continuum limit.

The main focus of this paper is to investigate the phase structure for $\mu_{45} \neq 0$, in particular to clarify, whether inhomogeneous phases exist. At first, we recall that the effective action $S_{\text {eff }}[\sigma]$ can be written as sum of a left-handed part $S_{\text {eff }}^{L}[\sigma]$ and a righthanded part $S_{\text {eff }}^{R}[\sigma]$ with chemical potentials $\mu_{L}$ and $\mu_{R}$, respectively (see Eq. (2.21)). Moreover, each of the two parts is equivalent to the action of the chirally balanced GN model, which was investigated in detail in our previous work [29]. Thus, for $\left|\mu_{L}\right|>\mu_{c}(T)$ and $\left|\mu_{R}\right|>\mu_{c}(T)$ both $S_{\text {eff }}^{L}[\sigma]$ and $S_{\text {eff }}^{R}[\sigma]$ have their respective minima at $\sigma=0\left(\mu_{c}(T)\right.$ denotes the location of the phase boundary of the symmetric phase at $\mu_{45}=0$ and temperature $\left.T\right)^{3}$. Consequently, the minimum of $S_{\text {eff }}[\sigma]$ also corresponds to $\sigma=0$. In other words, from numerical results obtained in Ref. [29] at $\mu_{45}=0$ we can conclude that chiral symmetry is restored in the chirally imbalanced GN model for $\left|\mu_{L}\right|>\mu_{c}(T)$ and $\left|\mu_{R}\right|>\mu_{c}(T)$. In the remaining regions of $\left(\mu, \mu_{45}, T\right)$ space $S_{\text {eff }}^{L}[\sigma]$ and $S_{\text {eff }}^{R}[\sigma]$ compete and the behavior of the condensate needs to be investigated numerically. We start doing that in Section 4.1 by restricting our computations to a homogeneous

3 We ignore the existence of an "inhomogeneous island" or "inhomogeneous continent" at large chemical potential, where cutoff effects are particularly strong [29]. 
condensate. After that, in Section 4.2, we carry out a stability analysis of the favored value of the homogeneous condensate with respect to inhomogeneous perturbations. Finally, in Section 4.3, we perform numerical minimizations of the effective action allowing arbitrary inhomogeneous modulations.

The lattice spacing $a$ is a function of the coupling $\lambda$. As explained in our previous work [29] we tune $\lambda$ such that the temporal extent $N_{t, c} a$ corresponds to the inverse critical temperature $\beta_{c}=1 / T_{c}$, which separates at $\mu=\mu_{45}=0$ the symmetric and the homogeneous symmetry-broken phase. Then, at fixed $\lambda$, the temperature $T=1 / N_{t} a$ can be changed in discrete steps by increasing or decreasing $N_{t}$. A summary of the lattice parameters, which were used to generate all following numerical results, is given in Table 1 . We note that throughout this section dimensionful quantities are expressed in units of the vacuum expectation value of $\sigma$,

$$
\sigma_{0}=\left.\sigma\right|_{\mu=0, \mu_{45}=0, T=0}
$$

\begin{tabular}{cccc}
\hline$N_{t, c}$ & $\lambda / a$ & $a \sigma_{0}$ & $N_{s}$ \\
\hline 4 & 2.6040 & 0.3649 & $28,40,60,80$ \\
6 & 2.3355 & 0.2327 & $60,100,120$ \\
\hline
\end{tabular}

Table 1: Lattice parameters $\left(N_{t, c}:\right.$ number of lattice sites in temporal direction corresponding to the critical temperature $T_{c} ; \lambda$ : coupling; $a$ : lattice spacing; $N_{\mathcal{S}}$ : number of lattice sites in each of the two spatial directions).

\subsection{Restriction to a homogeneous condensate}

In the case of a homogeneous condensate, i.e., $\sigma=\bar{\sigma}$ or equivalently $\tilde{\sigma}(\mathbf{p})=\bar{\sigma} \delta_{\mathbf{p}, 0}$, the two lattice discretizations with $\tilde{W}_{2}^{\prime}$ and $\tilde{W}_{2}^{\prime \prime}$ (Eqs. (3.6) and (3.7)) are identical. The Dirac operator corresponds to a block-diagonal matrix with $N_{t} N_{s}^{2}$ blocks of size $4 \times 4$. Thus, the $\ln \operatorname{Det} \tilde{Q}^{(4)}\left[\mu, \mu_{45}, \sigma\right]$ term in the effective action (3.2) can be computed quite efficiently by summing over $N_{t} N_{s}^{2}$ determinants of $4 \times 4$ matrices. Moreover, the effective action at given $\mu, \mu_{45}$ and $T$ is a function of just a single variable $\bar{\sigma}$ and, hence, can be minimized numerically in a straightforward and rather cheap way to obtain the physically preferred value of the homogeneous condensate.

Fig. 1 shows the phase diagram in $\left(\mu, \mu_{45}, T\right)$ space for $a \sigma_{0}=0.2327$ and $L \sigma_{0}=120 a \sigma_{0}=27.92$. For $\mu_{45}=0.0$ the phase boundary is quite similar to the analytically obtained continuum result [47] with slight deviations due to discretization and finite volume effects. At large temperature $T / \sigma_{0} \gtrsim 0.4$ the phase boundary exhibits an approximate rotational symmetry in the $\mu-\mu_{45}$ plane, i.e. is crudely described by $\mu^{2}+\mu_{45}^{2} \approx\left(\mu_{c}(T)\right)^{2}$. In contrast to that, at small temperature the phase boundary approaches a square-like shape in the $\mu-\mu_{45}$ plane.

The left plot of Fig. 2 shows sectional views of the phase diagram at fixed lattice spacing $a \sigma_{0}=0.2327$ for two different spatial extents, $L \sigma_{0}=60 a \sigma_{0}=13.95$ and $L \sigma_{0}=120 a \sigma_{0}=27.92$. At small temperature the phase boundary exhibits an oscillatory behavior, which is more pronounced for the smaller lattice volume. We expect that the oscillations disappear in the infinite volume limit. The right plot of Fig. 2 shows sectional views of the phase diagram for two different lattice spacings, $a \sigma_{0}=0.3649$ and $a \sigma_{0}=$ 0.2327 , at fixed ratio $N_{s} / N_{t, c}=20$ implying similar spatial extents $L \sigma_{0}=80 a \sigma_{0}=29.19$ and $L \sigma_{0}=120 a \sigma_{0}=27.92$. There are visible discrepancies due to discretization effects, in particular, when both $T$ is small and $\mu \approx \mu_{45}$. Continuum results at $\mu_{45}=0$ from Ref. [47] as well as our lattice results at various small temperatures, lattice spacings and spatial volumes point towards $T=0$ phase boundaries at $\mu / \sigma_{0}=1$ for $0 \leq \mu_{45} / \sigma_{0}<1$ and at $\mu_{45} / \sigma_{0}=1$ for $0 \leq \mu / \sigma_{0}<1$ in the continuum limit.

We have also studied the behavior of $\bar{\sigma}$ at small temperature $T / \sigma_{0}=0.0716$, $a \sigma_{0}=0.2327$ and $L \sigma_{0}=120 a \sigma_{0}=27.92 . \bar{\sigma}$ is shown as function of $\mu$ and $\mu_{45}$ in the 


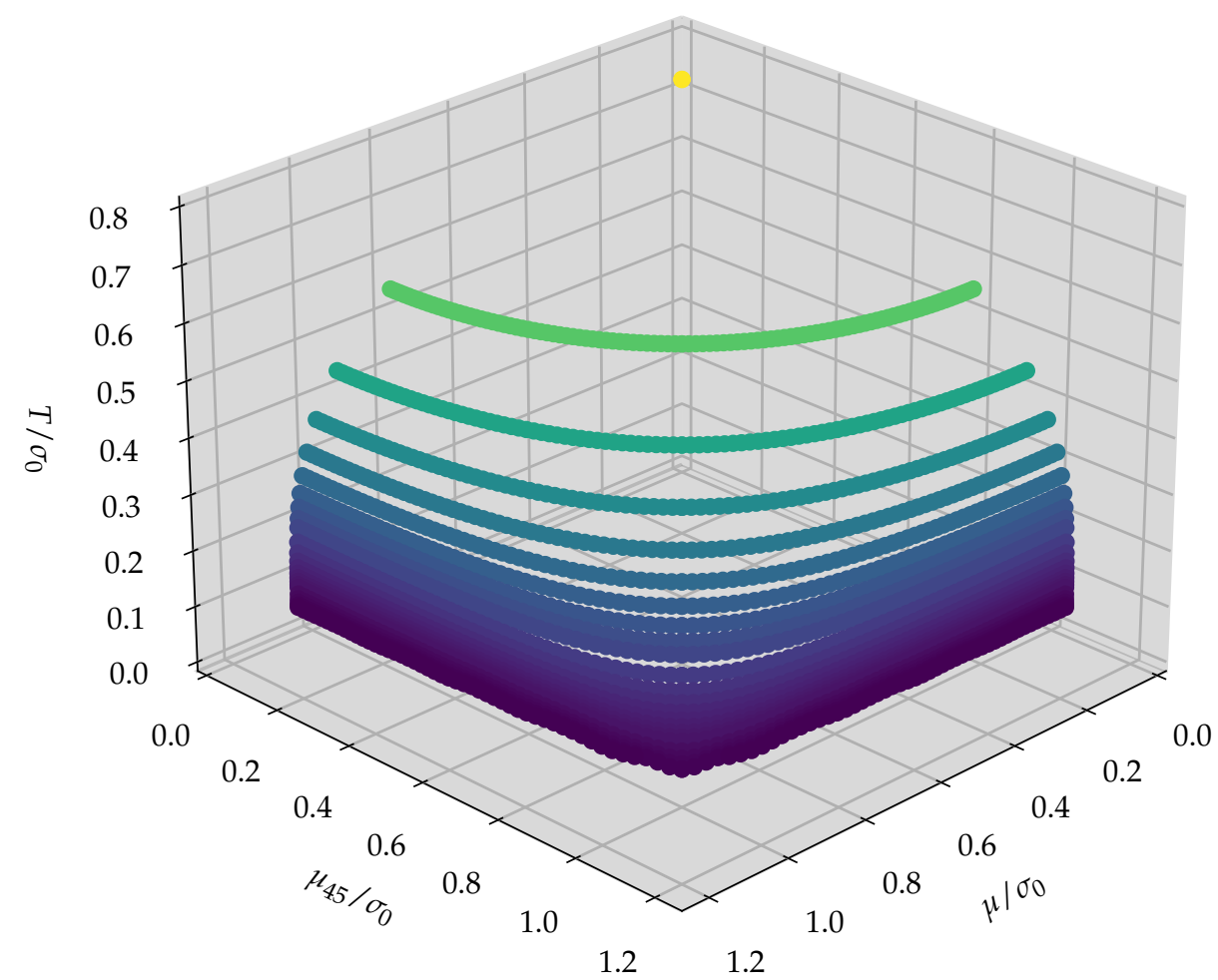

Figure 1. Phase diagram of the chirally imbalanced $2+1$-dimensional GN model with the restriction to a homogeneous condensate $\sigma=\bar{\sigma}$ in $\left(\mu, \mu_{45}, T\right)$ space for $a \sigma_{0}=0.2327$ and $L \sigma_{0}=120 a \sigma_{0}=27.92$.
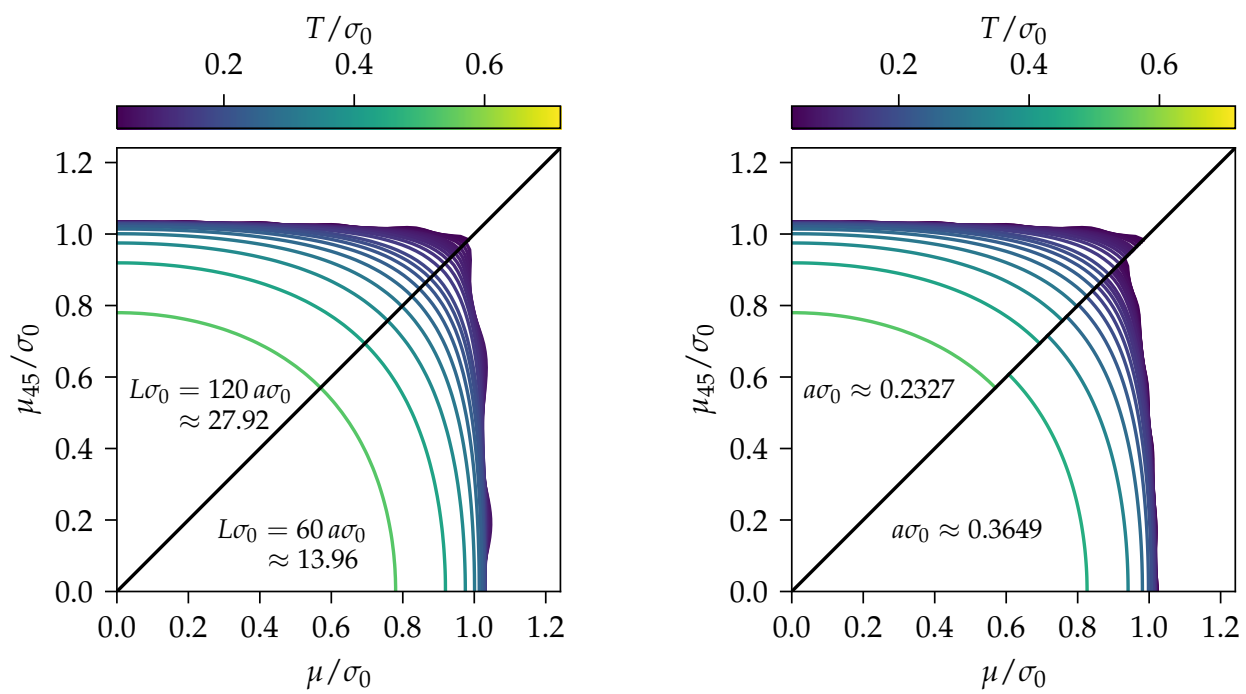

Figure 2. Phase diagram of the chirally imbalanced $2+1$-dimensional GN model with the restriction to a homogeneous condensate $\sigma=\bar{\sigma}$ in the $\mu-\mu_{45}$ plane for several temperatures. Since the phase diagram is invariant with respect to $\mu \leftrightarrow \mu_{45}$, each octant in the $\mu$ - $\mu_{45}$ plane contains the full information and one can compare two lattice extents $L \sigma_{0}$ (left plot) or two lattice spacings $a \sigma_{0}$ (right plot) in a convenient way within the same plot. (left) $a \sigma_{0}=0.2327$. (right) $N_{s} / N_{t, c}=20$, i.e., similar spatial lattice extents $L \sigma_{0}=80 a \sigma_{0}=29.19$ and $L \sigma_{0}=120 a \sigma_{0}=27.92$. 

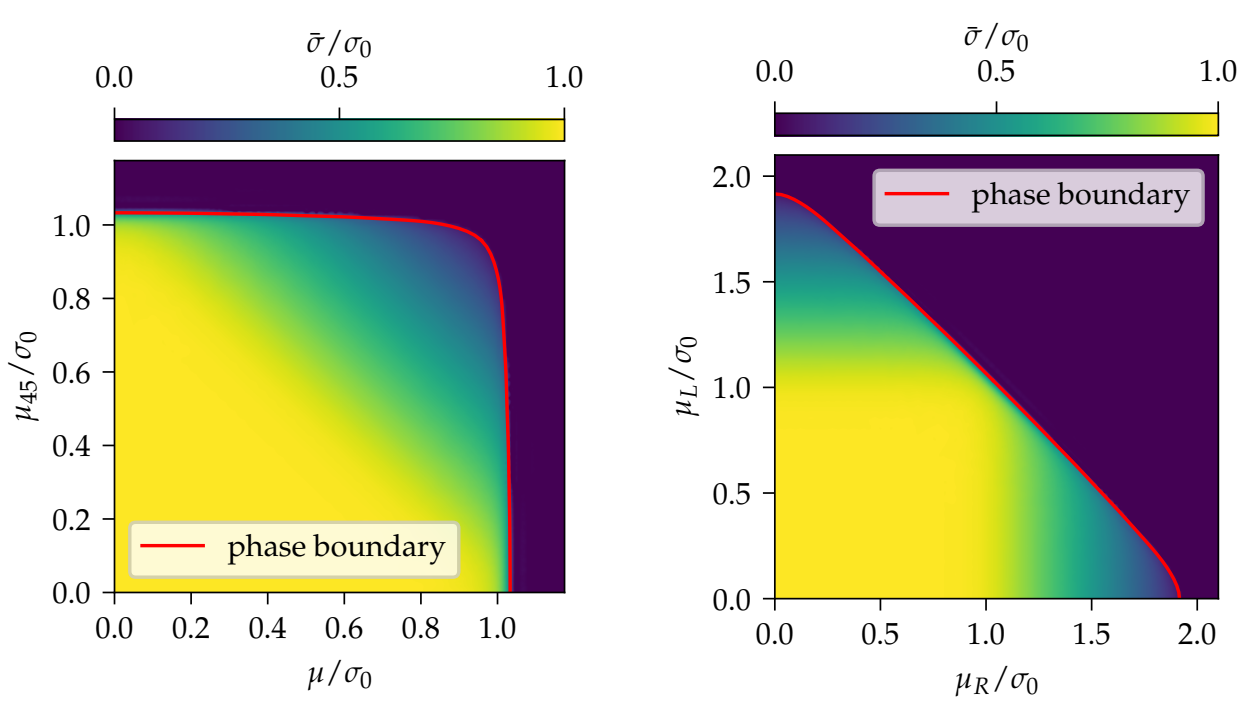

Figure 3. $\bar{\sigma} / \sigma_{0}$ for the chirally imbalanced $2+1$-dimensional GN model with the restriction to a homogeneous condensate $\sigma=\bar{\sigma}$ for $T / \sigma_{0}=0.0716, a \sigma_{0}=0.2327$ and $L \sigma_{0}=120 a \sigma_{0}=27.92$. (left) $\bar{\sigma} / \sigma_{0}$ as function of $\mu$ and $\mu_{45}$. (right) $\bar{\sigma} / \sigma_{0}$ as function of $\mu_{L}$ and $\mu_{R}$.

left plot of Fig. 3 and as function of $\mu_{L}=\mu+\mu_{45}$ and $\mu_{R}=\mu-\mu_{45}$ in the right plot of Fig. 3. To explain these results, we note that the effective action (2.21) is the sum of a left-handed part $S_{\text {eff }}^{L}[\sigma]$ and a right-handed part $S_{\text {eff }}^{R}[\sigma]$ with chemical potentials $\mu_{L}$ and $\mu_{R}$, respectively. At the beginning of Section 4 we already concluded that $\bar{\sigma}=0$, if $\left|\mu_{L}\right|>\mu_{c}(T)$ and $\left|\mu_{R}\right|>\mu_{c}(T)$. Similarly, we argue now that both parts favor $\bar{\sigma} \approx \sigma_{0}$, if $\left|\mu_{L}\right|<\mu_{c}(T)$ and $\left|\mu_{R}\right|<\mu_{c}(T)$. The numerical results from Fig. 3 are consistent with that expectation. In particular the yellow regions, where $\bar{\sigma} \approx \sigma_{0}$, correspond to $\left|\mu_{L}\right|<\mu_{c}(T)$ and $\left|\mu_{R}\right|<\mu_{c}(T)$. In the remaining regions of the $\mu-\mu_{45}$ plane, or equivalently the $\mu_{L}-\mu_{R}$ plane, $S_{\text {eff }}^{L}[\sigma]$ and $S_{\text {eff }}^{R}[\sigma]$ compete, leading to a continuous transition of the condensate from $\bar{\sigma} \approx \sigma_{0}$ to $\bar{\sigma}=0$. This continuous behavior is consistent with the fact that the lattice GN model with the effective action $\left.S_{\text {eff }}^{L}[\sigma]\right|_{\mu_{L}=\mu}$ or equivalently $\left.S_{\text {eff }}^{R}[\sigma]\right|_{\mu_{R}=\mu}$, restricted to a homogeneous condensate, has a second order phase transition at $T / \sigma_{0}=0.0716$, $a \sigma_{0}=0.2327$ and $L \sigma_{0}=120 a \sigma_{0}=27.92$.

We note that the lattice data shown in this subsection also represents a non-trivial cross-check of our implementation: all numerical results are consistent with the symmetry $\mu \leftrightarrow \mu_{45}$ within machine precision.

\subsection{Stability of a homogeneous condensate}

Now we relax the constraint that $\sigma$ is a homogeneous condensate. To determine the preferred modulation of the condensate in a possibly existing inhomogeneous phase, one has to allow arbitrary spatial modulations of $\sigma$, i.e., consider $\sigma=\sigma(\mathbf{x})$, and minimize the effective action with respect to these modulations. In lattice field theory this is possible, but numerically very challenging. In a first step, we, therefore, explore, whether the homogeneous minima $\sigma=\bar{\sigma}$, which were determined in Section 4.1 for many different $\left(\mu, \mu_{45}, T\right)$, are stable or unstable with respect to spatially inhomogeneous perturbations $\delta \sigma(\mathbf{x})$. Boundaries between stable and unstable regions in $\left(\mu, \mu_{45}, T\right)$ space are identical to phase boundaries, if the amplitude of the inhomogeneity becomes infinitesimal, when approaching the boundary. However, a stability analysis fails to detect inhomogeneous condensates in regions of the phase diagram, where the homogeneous minimum (found, e.g., as described in Section 4.1) corresponds to a local, but not to a global minimum of $S_{\text {eff }}[\sigma(\mathbf{x})] / N_{f}$. This is, e.g., the case in the 1+1-dimensional GN model [26,63].

A detailed derivation of the formalism to probe the stability of a homogeneous condensate $\sigma=\bar{\sigma}$ with respect to arbitrary spatial perturbations $\delta \sigma(\mathbf{x})$ can be found 

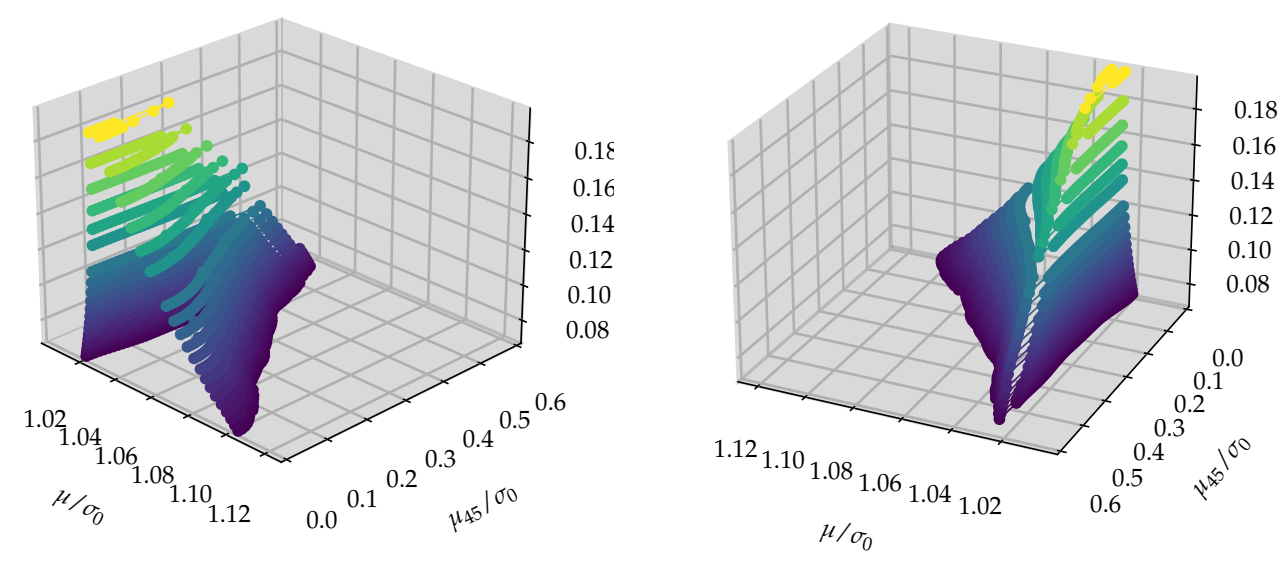

Figure 4. Boundaries of the region of instability in the chirally imbalanced $2+1$-dimensional GN model for the discretization $\tilde{W}_{2}=\tilde{W}_{2}^{\prime \prime}$ in $\left(\mu, \mu_{45}, T\right)$ space for $a \sigma_{0}=0.2327$ and

$L \sigma_{0}=100 a \sigma_{0}=23.27$. Both plots show the same data and differ only in the angle of view.

in Ref. [29] for a continuum approach. This formalism can be transferred to lattice discretizations in a straightforward way, which is discussed in the same reference. A quantity of central importance is

$$
\frac{\Gamma^{-1}\left(\mathbf{q}_{k}\right)}{N_{f}}=\frac{1}{\lambda}-\frac{\tilde{W}_{2}\left(\mathbf{q}_{k}\right) \tilde{W}_{2}\left(-\mathbf{q}_{k}\right)}{8} \sum_{p} \operatorname{tr}\left(\bar{Q}_{p-q}^{-1}\left[\mu, \mu_{45}, \bar{\sigma}\right] \bar{Q}_{p}^{-1}\left[\mu, \mu_{45}, \bar{\sigma}\right]\right),
$$

where $\sum_{p}$ runs over all 3-dimensional lattice momenta (3.1), $q=\left(0, \mathbf{q}_{k}\right)$, the trace refers to spinor space and $\bar{Q}_{p}\left[\mu, \mu_{45}, \bar{\sigma}\right]$ is defined via $\tilde{Q}_{p, q}^{(4)}\left[\mu, \mu_{45}, \bar{\sigma}\right]=\delta_{p, q} \bar{Q}_{p}\left[\mu, \mu_{45}, \bar{\sigma}\right]$ and Eq. (3.4), i.e.,

$$
\bar{Q}_{p}\left[\mu, \mu_{45}, \bar{\sigma}\right]=N_{t} N_{s}^{2}\left(\mathrm{i} \sum_{v=0}^{2} \gamma_{v} \sin \left(p_{v}-\delta_{v, 0} \mathrm{i}\left(\mu+\gamma_{45} \mu_{45}\right)\right)+\bar{\sigma}\right) .
$$

Negative values of $\Gamma^{-1}\left(\mathbf{q}_{k}\right) / N_{f}$ with $\mathbf{q}_{k} \neq 0$ indicate instability of the condensate $\sigma=\bar{\sigma}$ with respect to harmonic perturbations with momentum $\mathbf{q}_{k}$. Such perturbations decrease $S_{\text {eff }}[\sigma]$ and, consequently, an inhomogeneous condensate is preferred. By evaluating $\Gamma^{-1}\left(\mathbf{q}_{k}\right) / N_{f}$ for suitably chosen parameters $\left(\mu, \mu_{45}, T\right)$ one can identify regions, which are part of an inhomogeneous phase.

We searched extensively for regions, where $\bar{\sigma}$ is unstable, using both discretizations (3.6) and (3.7). For $\tilde{W}_{2}=\tilde{W}_{2}^{\prime}$ such regions do not seem to exist. For $\tilde{W}_{2}=\tilde{W}_{2}^{\prime \prime}$ and finite lattice spacing there is a region of instability at small $T$ consistent with the findings at $\mu_{45}=0$ reported in Ref. [29]. It is shown in Fig. 4 for the lattice with the finer lattice spacing, $a \sigma_{0}=0.2327$, and spatial extent $L \sigma_{0}=100 a \sigma_{0}=23.27$. The region of instability is located within the tetrahedral shape. At smaller temperature it has a larger extent in the $\mu-\mu_{45}$ plane. A somewhat unexpected result is the large extent of the region of instability in $\mu_{45}$ direction, e.g., for $T / \sigma_{0}=0.076$ and $\mu / \sigma_{0} \approx 1.0$ up to $\mu_{45} / \sigma_{0} \approx 0.5$. Its boundary is plotted in Fig. 5 in the $\mu_{R}-\mu_{L}$ plane. The plot shows that the instability region extends up to $\mu_{L}=\mu+\mu_{45} \approx 1.5$ and at the same time down to $\mu_{R}=\mu-\mu_{45} \approx 0.5$. A symmetric phase is preferred by $S_{\text {eff }}^{L}[\sigma]$ with chemical potential $\mu_{L} \approx 1.5$, while $S_{\text {eff }}^{R}[\sigma]$ with chemical potential $\mu_{R} \approx 0.5$ prefers a homogeneous symmetry-broken phase. Thus, neither of two parts of the effective action (2.21) favors an inhomogeneous phase, but in combination they do. This highlights the non-trivial interplay of $S_{\mathrm{eff}}^{L}[\sigma]$ and $S_{\mathrm{eff}}^{R}[\sigma]$ giving rise to a rather large inhomogeneous phase at finite lattice spacing for certain discretizations. Note that Fig. 5 also reveals that the homogeneous phase boundary 
is engulfed by the region of instability, which is not the case in our previous study at $\mu_{45}=0$ [29], where a different lattice regularization was used.

In Fig. 6 we show sectional views of the region of instability for the discretization $\tilde{W}_{2}=\tilde{W}_{2}^{\prime \prime}$ and various temperatures. The upper row corresponds to the larger lattice spacing, $a \sigma_{0}=0.3649$, and the lower row to the smaller lattice spacing, $a \sigma_{0}=0.2327$, the left column to smaller and the the right column to larger spatial extent $L \sigma_{0}$. Comparing the upper and the lower row it is obvious that the instability region shrinks, when decreasing the lattice spacing, most prominently in $\mu$ direction. This is particularly evident from the right column, where the boundaries are significantly less distorted by finite volume effects. In the plots in the left column, however, one can see pronounced oscillations, which seem to be caused by small spatial volume. These oscillations are reminiscent of those observed in the $\mu$-T plane in lattice studies of the chirally balanced GN model in $1+1$ and $2+1$ dimensions [27,29,63].

In summary, we found no region of instability for $\tilde{W}_{2}=\tilde{W}_{2}^{\prime}$ and a shrinking region of instability for decreasing lattice spacing for $\tilde{W}_{2}=\tilde{W}_{2}^{\prime \prime}$. This indicates that there is no region of instability in the chirally imbalanced $2+1$-dimensional GN model in the continuum limit.

\subsection{Arbitrary spatial modulations of the condensate}

Now we discuss the minimization of the effective action (3.2) with respect to the condensate allowing arbitrary spatial modulations, i.e. arbitrary Fourier coefficients $\tilde{\sigma}(\mathbf{p})$. We did this for selected parameters $\left(\mu, \mu_{45}, T\right)$ by carrying out several conjugate gradient minimizations, which differ in the starting values for $\tilde{\sigma}(\mathbf{p})$. For each $\left(\mu, \mu_{45}, T\right)$ we found only a small number of local minima, although a significantly larger number of different starting values for $\tilde{\sigma}(\mathbf{p})$ were provided to the minimization algorithm. This might indicate that for all considered $\left(\mu, \mu_{45}, T\right)$ the corresponding global minimum is among the found local minima. We note that in our previous work [29] only 1-dimensional modulations were studied, i.e., $\tilde{\sigma}=\tilde{\sigma}\left(p_{1}\right)$. In this work we relax that constraint and allow arbitrary 2-dimensional modulations, i.e., $\tilde{\sigma}=\tilde{\sigma}(\mathbf{p})$. Since this is a numerically difficult and computer time intensive task, we use a rather small lattice with coarse lattice spacing $a \sigma_{0}=0.3649$, temperature $T / \sigma_{0}=0.114, L \sigma_{0}=28 a \sigma_{0}=10.22$ and the discretization corresponding to $\tilde{W}_{2}=\tilde{W}_{2}^{\prime \prime}$.

In a first step, we studied the chirally balanced model, i.e. $\mu_{45}=0$. As an example, the upper left plot in Fig. 7 shows a configuration $\sigma(\mathbf{x})$ corresponding to one of the global minima of the effective action at $\mu / \sigma_{0}=1.041$. An inhomogeneous condensate is favored, as we know from the stability analysis discussed in Section 4.2. Even though the minimization algorithm allows arbitrary 2-dimensional modulations, the resulting global minimum is just a plane wave with wave vector $\mathbf{q}_{k}=2 \pi(1,2) / \mathrm{L}$. Similarly, the upper right plot in Fig. 7 shows a minimizing condensate at larger chemical potential $\mu / \sigma_{0}=1.083$. Again we found a plane wave, this time with wave vector $\mathbf{q}_{k}=2 \pi(2,2) / L$, i.e., with a smaller wavelength. Even though the found plane waves are 1-dimensional structures, allowing arbitrary 2-dimensional modulations reduces finite volume corrections. This is so, because, in contrast to our previous work [29], the direction of the wave vector is not anymore restricted to be parallel to one of the coordinate axes and, thus, its magnitude can now be changed in finer steps.

The minimization algorithm also found 2-dimensional modulations. These, however, correspond exclusively to local minima of the effective action (3.2). An example is shown in the center of Fig. 7.

We also investigated, how chiral imbalance, i.e. $\mu_{45} \neq 0$, affects the preferred modulation of the condensate. The plots in the lower row of Fig. 7 show global minima of the effective action for $\left(\mu / \sigma_{0}, \mu_{45} / \sigma_{0}\right)=(1.041,0.05)$ and $\left(\mu / \sigma_{0}, \mu_{45} / \sigma_{0}\right)=(1.041,0.25)$. At fixed $\mu / \sigma_{0}=1.041$ the frequency is almost independent of $\mu_{45}$ (cf. the upper left plot, the lower left plot and the lower right plot of Fig. 7). The amplitude, however, decreases, when increasing $\left|\mu_{45}\right|$, supporting a second order phase transition also at 


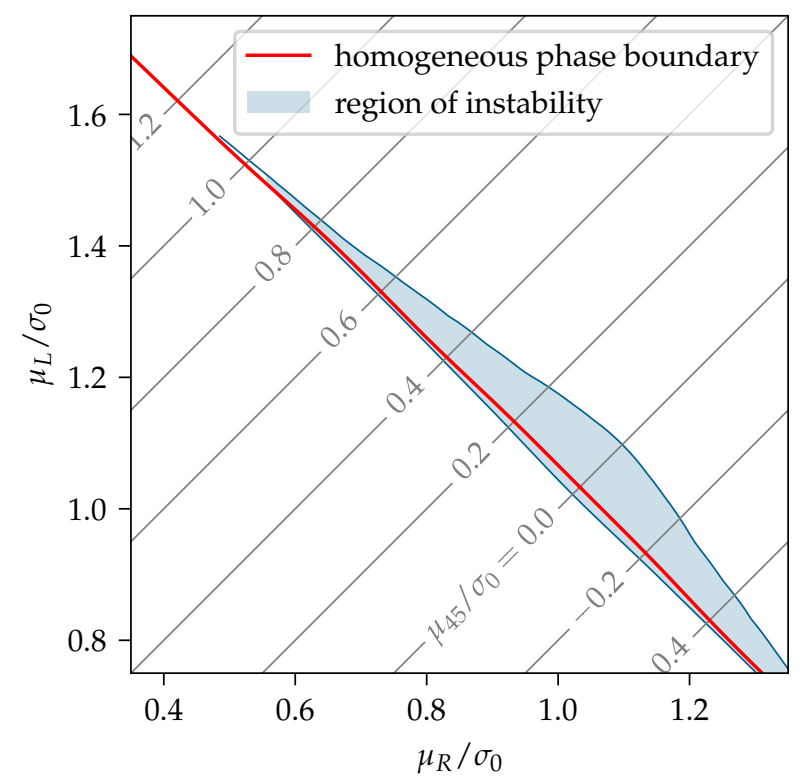

Figure 5. Region of instability and homogeneous phase boundary in the chirally imbalanced $2+1$-dimensional GN model for the discretization $\tilde{W}_{2}=\tilde{W}_{2}^{\prime}$ in the $\mu_{R}-\mu_{L}$ plane for $T / \sigma_{0}=0.076$, $a \sigma_{0}=0.2327$ and $L \sigma_{0}=100 a \sigma_{0}=23.27$. The chiral chemical potential $\mu_{45}$ is constant along the diagonal straight lines.

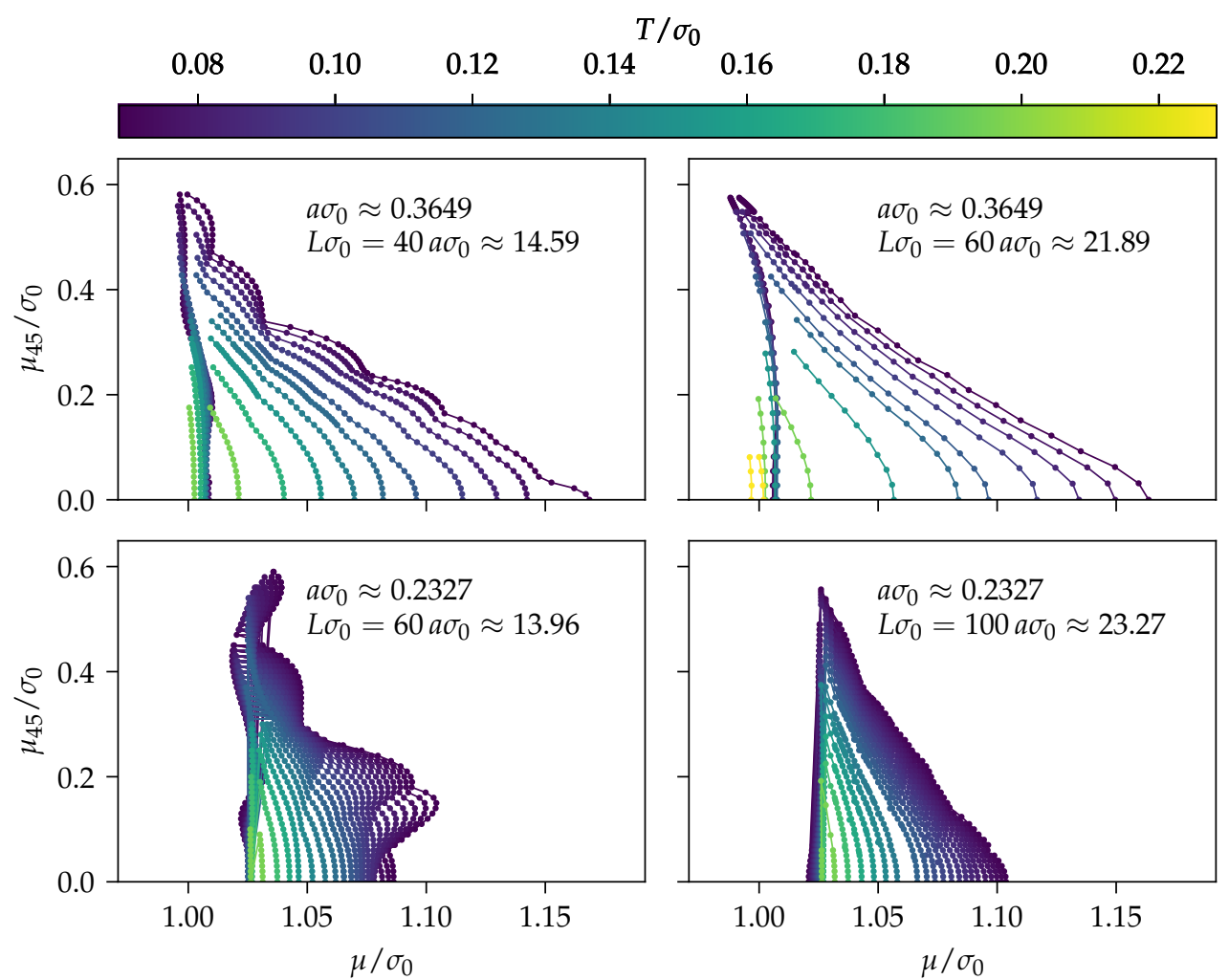

Figure 6. Boundaries of the region of instability in the chirally imbalanced $2+1$-dimensional GN model for the discretization $\tilde{W}_{2}=\tilde{W}_{2}^{\prime \prime}$ in the $\mu-\mu_{45}$ plane for several temperatures, two different $a \sigma_{0}$ (upper versus lower row) and two different $L \sigma_{0}$ (left versus right column). 
1-dimensional modulations corresponding to global minima, $\mu_{45}=0$
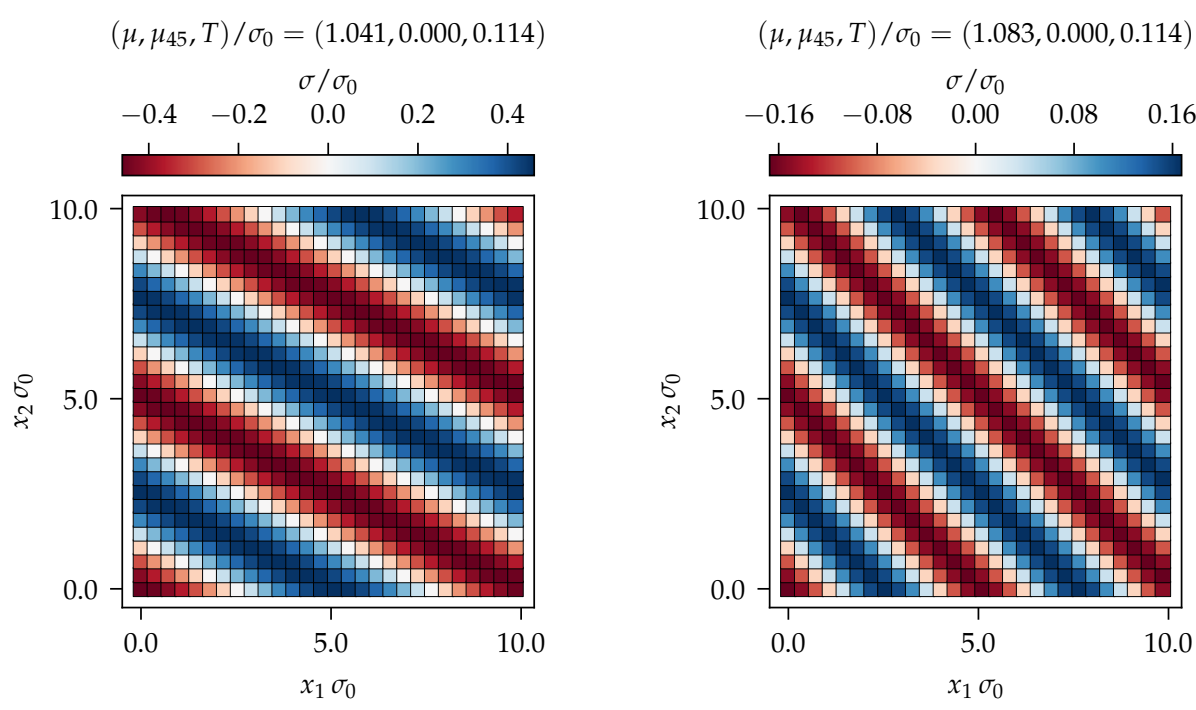

2-dimensional modulation corresponding to a local minimum, $\mu_{45}=0$

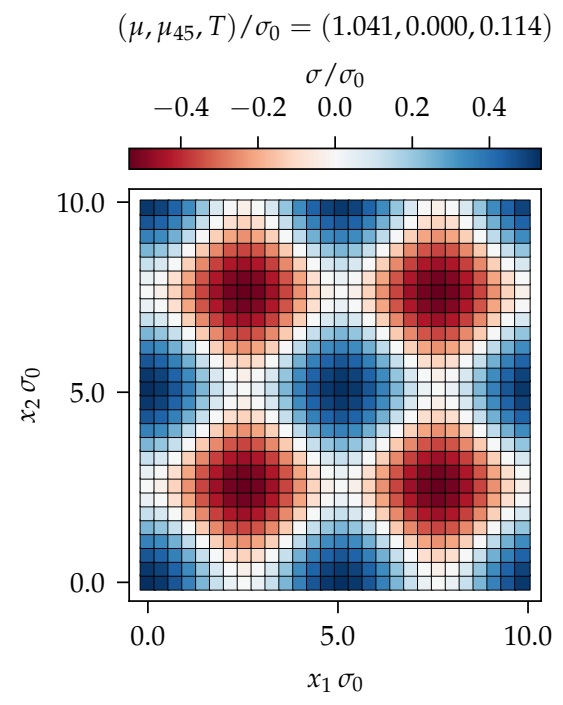

1-dimensional modulations corresponding to global minima, $\mu_{45} \neq 0$
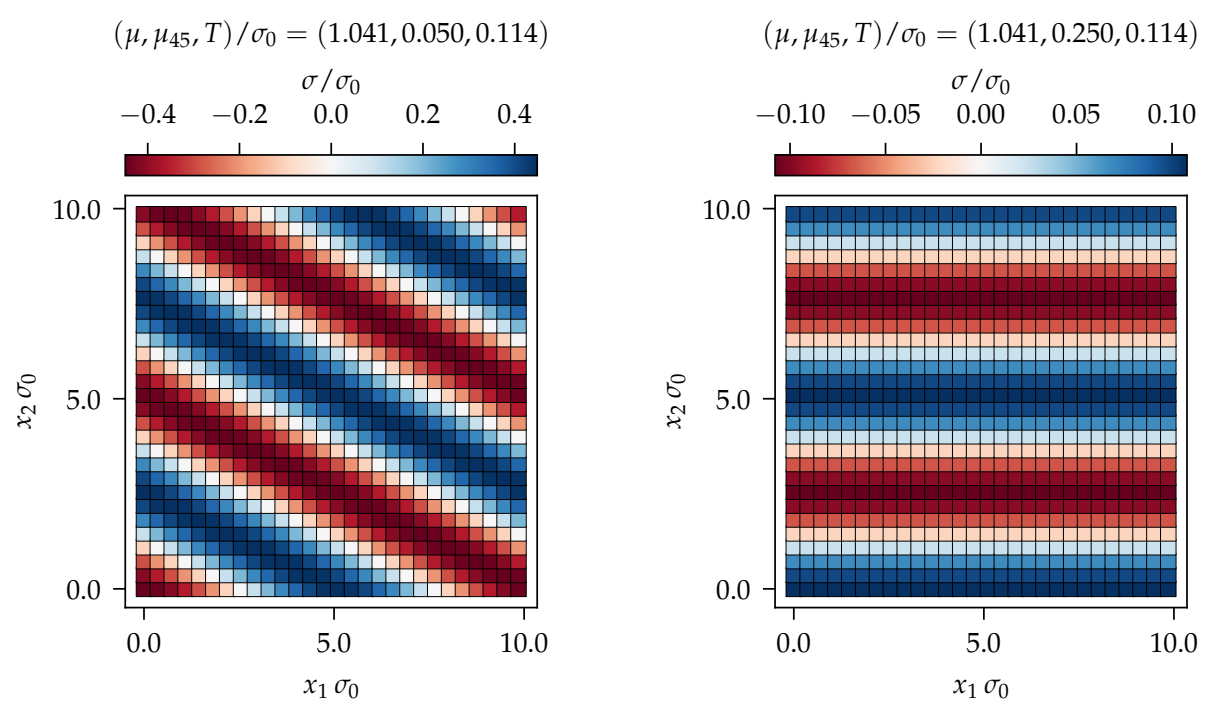

Figure 7. Modulations of the condensate $\sigma$ corresponding to minima of the effective action (3.2) for the discretization $\tilde{W}_{2}=\tilde{W}_{2}^{\prime \prime}, T / \sigma_{0}=0.114, a \sigma_{0}=0.3649$ and $L \sigma_{0}=28 a \sigma_{0}=10.22$. 
$\mu_{45} \neq 0$. We searched extensively for inhomogeneous condensates outside the instability region explored and discussed in Section 4.2, but did not find any. Thus, we conclude that the boundaries of the instability region are identical to phase boundaries and that the corresponding phase transitions are of second order.

\section{Conclusions}

In this work we have studied the phase diagram of the $2+1$-dimensional GN model with chiral imbalance introduced via a chiral chemical potential $\mu_{45}$. Our lattice field theory results indicate that an inhomogeneous phase exists at finite lattice spacing $a$, when using a specific lattice discretization $\left(\tilde{W}_{2}=\tilde{W}_{2}^{\prime}\right)$. Non-vanishing $\mu_{45}$, however, seems to disfavor inhomogeneous modulations. Moreover, the inhomogeneous phase shrinks for decreasing $a$ and is expected to disappear in the continuum limit. These findings are consistent with our previous work [29] restricted to $\mu_{45}=04$.

Moreover, we have shown for our chirally imbalanced $2+1$-dimensional GN model that an isospin chemical potential $\mu_{\mathrm{I}}$ is equivalent to the chiral chemical potential $\mu_{45}$. Thus, all results presented can either be interpreted in the context of chiral imbalance or of isospin imbalance. In particular the $\mu-\mu_{45^{-}}-T$ phase diagram is identical to the $\mu-\mu_{I}-T$ phase diagram. Interestingly, a recent study of the $3+1$-dimensional NJL model in the large- $N_{c}$ limit [53] conjectures a similar approximate duality of the phase diagram.

At finite isospin chemical potential color-superconductivity might play an important role. In the considered $2+1$-dimensional GN model this can, however, not be investigated, because the necessary difermion interaction is not present. Thus, we are not yet in a position to compare our results to up-to-date lattice QCD simulations at finite $\mu_{\mathrm{I}}$ (see, e.g., Refs. [65-69]), where a phase with Bose-Einstein condensation of charged pions was observed. In a next step, it might, thus, be interesting to establish contact to Refs. [56,57], where a color-superconducting channel was added to the chirally imbalanced $2+1$-dimensional GN model. It should, however, be noted that these references introduce the chiral chemical potential in a conceptually different way using spin matrices $\gamma_{0} \gamma_{4}$ as well as $\gamma_{0} \gamma_{5}$, instead of $\gamma_{0} \gamma_{45}$ appearing in Eq. (2.18).

Author Contributions: All authors contributed equally to this work. All authors have read and agreed to the published version of the manuscript.

Funding: L.P., M.Wa. and M.Wi. acknowledge support by the Deutsche Forschungsgemeinschaft (DFG, German Research Foundation) through the CRC-TR 211 "Strong-interaction matter under extreme conditions" - project number 315477589 - TRR 211. L.P. and M.W. acknowledge the support of the Helmholtz Graduate School for Hadron and Ion Research. M.Wa. acknowledges support by the Heisenberg Programme of the Deutsche Forschungsgemeinschaft (DFG, German Research Foundation) - project number 399217702. M.Wi. acknowledges support by the GSI Forschungs- und Entwicklungsvereinbarungen (GSI F\&E). M.Wi. acknowledges the support of the Giersch Foundation.

Data Availability Statement: The data set is included in the source files of the pre-print uploaded on arXiv.

Acknowledgments: We thank M. Buballa, A. Königstein, L. Kurth, A. Sciarra and M. Thies for valuable discussions regarding this work. We further thank J. Lenz, M. Mandl, R.D. Pisarksi and A. Wipf for general valuable discussions regarding inhomogeneous phases and four-fermion theories.

Numerical results in this work were produced with a C++ code using the nlopt library [70] for numerical minimization and the eigen3 library for matrix operations [71]. The data used in the figures were processed with the pandas [72] and numpy [73] packages. All figures in this work were produced with the matplotlib package [74].

4 For completeness we note that inhomogeneous condensates at $T=0$ and $\mu_{45}=0$, which are energetically degenerate to the homogenous condensate in the homogeneous symmetry-broken phase, were found in Ref. [64]. Our numerical lattice studies are, however, limited to $T>0$. 
Calculations on the GOETHE-HLR and on the FUCHS-CSC high-performance computer of the Frankfurt University were conducted for this research. We would like to thank HPC-Hessen, funded by the State Ministry of Higher Education, Research and the Arts, for programming advice.

Conflicts of Interest: The authors declare no conflict of interest.

\section{References}

1. Gross, D.J.; Neveu, A. Dynamical Symmetry Breaking in Asymptotically Free Field Theories. Phys. Rev. 1974, D10, 3235. doi:10.1103/PhysRevD.10.3235.

2. Thies, M.; Urlichs, K. Revised Phase Diagram of the Gross-Neveu Model. Phys. Rev. 2003, D67, 125015, [hep-th/0302092]. doi:10.1103/PhysRevD.67.125015.

3. Schnetz, O.; Thies, M.; Urlichs, K. Phase Diagram of the Gross-Neveu Model: Exact Results and Condensed Matter Precursors. Annals Phys. 2004, 314, 425-447, [hep-th/0402014]. doi:10.1016/j.aop.2004.06.009.

4. Thies, M. From Relativistic Quantum Fields to Condensed Matter and Back Again: Updating the Gross-Neveu Phase Diagram. J. Phys. A: Math. Gen. 2006, 39, 12707-12734, [hep-th/0601049]. doi:10.1088/0305-4470/39/41/S04.

5. Cohen, Y.; Elitzur, S.; Rabinovici, E. A Monte Carlo Study of the Gross-Neveu Model. Nucl. Phys. 1983, B220, 102-118. doi:10.1016/0550-3213(83)90136-0.

6. Karsch, F.; Kogut, J.B.; Wyld, H.W. The Gross-Neveu Model at Finite Temperature and Density. Nucl. Phys. 1987, B280, 289-303. doi:10.1016/0550-3213(87)90149-0.

7. Lenz, J.; Pannullo, L.; Wagner, M.; Wellegehausen, B.; Wipf, A. Inhomogeneous Phases in the Gross-Neveu Model in $1+1$ Dimensions at Finite Number of Flavors. Phys. Rev. D 2020, 101, 094512. doi:10.1103/PhysRevD.101.094512.

8. Lenz, J.J.; Pannullo, L.; Wagner, M.; Wellegehausen, B.; Wipf, A. Baryons in the Gross-Neveu Model in 1+1 Dimensions at Finite Number of Flavors. Phys. Rev. D 2020, 102, 114501, [2007.08382]. doi:10.1103/PhysRevD.102.114501.

9. Lenz, J.J.; Mandl, M.; Wipf, A. Inhomogeneities in the 2-Flavor Chiral Gross-Neveu Model. Pre-print, 2021, [2109.05525].

10. Lenz, J.J.; Mandl, M. Remnants of Large- $N_{\mathrm{f}}$ Inhomogeneities in the 2-Flavor Chiral Gross-Neveu Model. Pre-print, 2021, [2110.12757].

11. Stoll, J.; Zorbach, N.; Koenigstein, A.; Steil, M.J.; Rechenberger, S. Bosonic Fluctuations in the (1+ 1)-Dimensional Gross-Neveu(Yukawa) Model at Varying $m u$ and T and Finite N. Pre-print, 2021, [2108.10616].

12. Basar, G.; Dunne, G.V.; Thies, M. Inhomogeneous Condensates in the Thermodynamics of the Chiral NJL(2) Model. Phys. Rev. 2009, D79, 105012, [0903.1868]. doi:10.1103/PhysRevD.79.105012.

13. Thies, M. Chiral Spiral in the Presence of Chiral Imbalance. Phys. Rev. D 2018, 98, 096019, [1810.03921]. doi:10.1103/PhysRevD.98.096019.

14. Thies, M. Phase Structure of the $1+1$ Dimensional Nambu-Jona-Lasinio Model with Isospin. Phys. Rev. D 2020, 101, 014010, [1911.11439]. doi:10.1103/PhysRevD.101.014010.

15. Thies, M. Twisted kink dynamics in multiflavor chiral Gross-Neveu model. J. Phys. A 2022, 55, 015401, [2106.03522]. doi:10.1088/1751-8121/ac3cde.

16. Nakano, E.; Tatsumi, T. Chiral Symmetry and Density Wave in Quark Matter. Phys. Rev. D 2005, 71, 114006, [hep-ph/0411350]. doi:10.1103/PhysRevD.71.114006.

17. Nickel, D. Inhomogeneous Phases in the Nambu-Jona-Lasino and Quark-Meson Model. Phys. Rev. 2009, D80, 074025, [0906.5295]. doi:10.1103/PhysRevD.80.074025.

18. Carignano, S.; Buballa, M.; Schaefer, B.J. Inhomogeneous Phases in the Quark-Meson Model with Vacuum Fluctuations. Phys. Rev. D 2014, 90, 014033, [1404.0057]. doi:10.1103/PhysRevD.90.014033.

19. Heinz, A.; Giacosa, F.; Wagner, M.; Rischke, D.H. Inhomogeneous Condensation in Effective Models for QCD Using the Finite-Mode Approach. Phys. Rev. 2016, D93, 014007, [1508.06057]. doi:10.1103/PhysRevD.93.014007.

20. Buballa, M.; Carignano, S. Inhomogeneous Chiral Condensates. Prog. Part. Nucl. Phys. 2015, 81, 39-96, [1406.1367]. doi:10.1016/j.ppnp.2014.11.001.

21. Pisarski, R.D.; Tsvelik, A.M.; Valgushev, S. How Transverse Thermal Fluctuations Disorder a Condensate of Chiral Spirals into a Quantum Spin Liquid. Phys. Rev. D 2020, 102, 016015, [2005.10259]. doi:10.1103/PhysRevD.102.016015.

22. Pisarski, R.D.; Rennecke, F.; Tsvelik, A.; Valgushev, S. The Lifshitz Regime and Its Experimental Signals. Nuclear Physics A 2021, 1005, 121910, [2005.00045]. doi:10.1016/j.nuclphysa.2020.121910.

23. Pisarski, R.D.; Rennecke, F. Signatures of Moat Regimes in Heavy-Ion Collisions. Phys. Rev. Lett. 2021, 127, 152302, [2103.06890]. doi:10.1103/PhysRevLett.127.152302.

24. Rennecke, F.; Pisarski, R.D. Moat Regimes in QCD and their Signatures in Heavy-Ion Collisions. International Conference on Critical Point and Onset of Deconfinement, 2021, [2110.02625].

25. Fu, W.j.; Pawlowski, J.M.; Rennecke, F. The QCD Phase Structure at Finite Temperature and Density. Phys. Rev. D 2020, 101, 054032, [1909.02991]. doi:10.1103/PhysRevD.101.054032.

26. Koenigstein, A.; Pannullo, L.; Rechenberger, S.; Winstel, M.; Steil, M.J. Detecting Inhomogeneous Chiral Condensation from the Bosonic Two-Point Function in the (1+1)-Dimensional Gross-Neveu Model in the Mean-Field Approximation. Pre-print, 2021, [2112.07024].

27. Winstel, M.; Stoll, J.; Wagner, M. Lattice investigation of an inhomogeneous phase of the $2+1$-dimensional Gross-Neveu model in the limit of infinitely many flavors. J. Phys. Conf. Ser. 2020, 1667, 012044, [1909.00064]. doi:10.1088/1742-6596/1667/1/012044. 
28. Narayanan, R. Phase diagram of the large N Gross-Neveu model in a finite periodic box. Phys. Rev. D 2020, 101, 096001, [2001.09200]. doi:10.1103/PhysRevD.101.096001.

29. Buballa, M.; Kurth, L.; Wagner, M.; Winstel, M. Regulator Dependence of Inhomogeneous Phases in the 2+1-Dimensional Gross-Neveu Model. Phys. Rev. D 2021, 103, 034503, [2012.09588]. doi:10.1103/PhysRevD.103.034503.

30. Hands, S.; Kocic, A.; Kogut, J. The Four-Fermi Model in Three Dimensions at Non-Zero Density and Temperature. Nuclear Physics B 1993, 390, 355-378, [hep-lat/9206024]. doi:10.1016/0550-3213(93)90460-7.

31. Hands, S.J.; Kogut, J.B.; Strouthos, C.G. The (2+1)-Dimensional Gross-Neveu Model with a U(1) Chiral Symmetry at Non-Zero Temperature. Physics Letters B 2001, 515, 407-413, [hep-lat/0107004]. doi:10.1016/S0370-2693(01)00885-1.

32. Gies, H.; Janssen, L. UV Fixed-Point Structure of the Three-Dimensional Thirring Model. Phys. Rev. D 2010, 82, 085018, [1006.3747]. doi:10.1103/PhysRevD.82.085018.

33. Scherer, D.D.; Gies, H. Renormalization Group Study of Magnetic Catalysis in the 3d Gross-Neveu Model. Phys. Rev. B 2012, 85, 195417, [1201.3746]. doi:10.1103/PhysRevB.85.195417.

34. Narayanan, R. The Relevance of the Three Dimensional Thirring Coupling at Finite Temperature and Density. Phys. Rev. D 2020, 102, 016014, [2006.00608]. doi:10.1103/PhysRevD.102.016014.

35. MacKenzie, R.; Panigrahi, P.K.; Sakhi, S. Superconductivity in a Planar Field Theory through the Kosterlitz-Thouless Mechanism. Phys. Rev. B 1993, 48, 3892-3895. doi:10.1103/PhysRevB.48.3892.

36. Tesanovic, Z.; Vafek, O.; Franz, M. Chiral Symmetry Breaking and Phase Fluctuations in Cuprate Superconductors: A QED 3 Unified Theory of the Pseudogap State. Phys. Rev. B 2002, 65, 180511, [cond-mat/0110253]. doi:10.1103/PhysRevB.65.180511.

37. Wen, X.G. Quantum Orders and Symmetric Spin Liquids. Phys. Rev. B 2002, 65, 165113, [cond-mat/0107071]. doi:10.1103/PhysRevB.65.165113.

38. Rantner, W.; Wen, X.G. Spin Correlations in the Algebraic Spin Liquid - Implications for High Tc Superconductors. Phys. Rev. B 2002, 66, 144501, [cond-mat/0201521]. doi:10.1103/PhysRevB.66.144501.

39. Ohsaku, T. Relativistic Model for Two-Band Superconductivity. Pre-print, 2003, [cond-mat/0306472].

40. Kalinkin, A.; Skorikov, V.M. Phase Transitions in Four Fermion Models. Inorg. Mater. 2003, 39, 765-779. doi:10.1023/A:1025063207931.

41. Hands, S.; Strouthos, C. Quantum Critical Behaviour in a Graphene-like Model. Phys. Rev. B 2008, 78, 165423, [0806.4877]. doi:10.1103/PhysRevB.78.165423.

42. Ziegler, L.; Tirrito, E.; Lewenstein, M.; Hands, S.; Bermudez, A. Correlated Chern Insulators in Two-Dimensional Raman Lattices: A Cold-Atom Regularization of Strongly-Coupled Four-Fermi Field Theories. Pre-print, 2020, [2011.08744].

43. Hands, S.; Kocic, A.; Kogut, J.B. Compositeness, Anomalous Dimensions and Renormalizability in Four Fermi Theories. Phys. Lett. B 1991, 273, 111-117. doi:10.1016/0370-2693(91)90562-5.

44. Wellegehausen, B.H.; Schmidt, D.; Wipf, A. Critical Flavor Number of the Thirring Model in Three Dimensions. Phys. Rev. 2017, D96, 094504, [1708.01160]. doi:10.1103/PhysRevD.96.094504.

45. Hands, S.; Mesiti, M.; Worthy, J. Critical Behaviour in the Single Flavor Thirring Model in 2+1d. Phys. Rev. D 2020, 102, 094502, [2009.02964]. doi:10.1103/PhysRevD.102.094502.

46. Hands, S. The Planar Thirring Model with K "ahler-Dirac Fermions. Symmetry 2021, 13, 1523, [2105.09646]. doi:10.3390/sym13081523.

47. Klimenko, K.G. Phase Structure of Generalized Gross-Neveu Models. Z. Phys. C 1988, 37, 457. doi:10.1007/BF01578141.

48. Rosenstein, B.; Warr, B.J.; Park, S.H. Thermodynamics of (2+1)-Dimensional Four Fermi Models. Phys. Rev. D 1989, $39,3088$. doi:10.1103/PhysRevD.39.3088.

49. Kharzeev, D.; Krasnitz, A.; Venugopalan, R. Anomalous Chirality Fluctuations in the Initial Stage of Heavy Ion Collisions and Parity Odd Bubbles. Physics Letters B 2002, 545, 298-306, [hep-ph/0109253]. doi:10.1016/S0370-2693(02)02630-8.

50. Kharzeev, D. Parity Violation in Hot QCD: Why It Can Happen, and How to Look for It. Physics Letters B 2006, 633, 260-264, [hep-ph/0406125]. doi:10.1016/j.physletb.2005.11.075.

51. Charbonneau, J.; Zhitnitsky, A. Topological Currents in Neutron Stars: Kicks, Precession, Toroidal Fields, and Magnetic Helicity. J. Cosmol. Astropart. Phys. 2010, 2010, 010-010, [0903.4450]. doi:10.1088/1475-7516/2010/08/010.

52. Ohnishi, A.; Yamamoto, N. Magnetars and the Chiral Plasma Instabilities 2014. [1402.4760].

53. Khunjua, T.G.; Klimenko, K.G.; Zhokhov, R.N. QCD Phase Diagram with Chiral Imbalance in NJL Model: Duality and Lattice QCD Results. J. Phys.: Conf. Ser. 2019, 1390, 012015, [1812.01392]. doi:10.1088/1742-6596/1390/1/012015.

54. Chernodub, M.N.; Ambrus, V.E. Phase Diagram of Helically Imbalanced QCD Matter. Phys. Rev. D 2021, 103, 094015, [2005.03575]. doi:10.1103/PhysRevD.103.094015.

55. Khunjua, T.G.; Klimenko, K.G.; Zhokhov, R.N. Influence of chiral asymmetry on phase structure of the two-color quark matter. Pre-print 2021, [2105.04952].

56. Ebert, D.; Khunjua, T.G.; Klimenko, K.G.; Zhukovsky, V.C. Competition and Duality Correspondence between Chiral and Superconducting Channels in (2+1)-Dimensional Four-Fermion Models with Fermion Number and Chiral Chemical Potentials. Phys. Rev. D 2016, 93, 105022, [1603.00357]. doi:10.1103/PhysRevD.93.105022.

57. Zhukovsky, V.C.; Klimenko, K.G.; Khunjua, T.G. Superconductivity in Chiral-Asymmetric Matter within the (2+1)-Dimensional Four-Fermion Model. Moscow Univ. Phys. 2017, 72, 250-256. doi:10.3103/S002713491703016X.

58. Braguta, V.V.; Goy, V.A.; Ilgenfritz, E.M.; Kotov, A.Y.; Molochkov, A.V.; Muller-Preussker, M.; Petersson, B. Two-Color QCD with Non-zero Chiral Chemical Potential. JHEP 2015, 06, 094, [1503.06670]. doi:10.1007/JHEP06(2015)094. 
59. Braguta, V.V.; Ilgenfritz, E.M.; Kotov, A.Y.; Petersson, B.; Skinderev, S.A. Study of QCD Phase Diagram with Non-Zero Chiral Chemical Potential. Phys. Rev. D 2016, 93, 034509, [1512.05873]. doi:10.1103/PhysRevD.93.034509.

60. Braguta, V.V.; Kotov, A.Y. Catalysis of Dynamical Chiral Symmetry Breaking by Chiral Chemical Potential. Phys. Rev. D 2016, 93, 105025, [1601.04957]. doi:10.1103/PhysRevD.93.105025.

61. Winstel, M.; Pannullo, L.; Wagner, M. Phase diagram of the 2+1-dimensional Gross-Neveu model with chiral imbalance. 38th International Symposium on Lattice Field Theory, 2021, [2109.04277].

62. Pisarski, R.D. Chiral Symmetry Breaking in Three-Dimensional Electrodynamics. Phys. Rev. D 1984, 29,2423 doi:10.1103/PhysRevD.29.2423.

63. de Forcrand, P.; Wenger, U. New Baryon Matter in the Lattice Gross-Neveu Model. Proceedings, 24th International Symposium on Lattice Field Theory (Lattice 2006): Tucson, USA, July 23-28, 2006, 2006, Vol. LAT2006, PoS, p. 152, [hep-lat/0610117]. doi:10.22323/1.032.0152.

64. Urlichs, K. Baryons and Baryonic Matter in Four-Fermion Interaction Models. PhD thesis, Friedrich-Alexander-Universität Erlangen-Nürnberg, 2007.

65. Brandt, B.B.; Endrodi, G. QCD phase diagram with isospin chemical potential. PoS 2016, LATTICE2016, 039, [1611.06758]. doi:10.22323/1.256.0039.

66. Brandt, B.B.; Cuteri, F.; Endrődi, G.; Schmalzbauer, S. The Dirac spectrum and the BEC-BCS crossover in QCD at nonzero isospin asymmetry. Particles 2020, 3, 80-86, [1912.07451]. doi:10.3390/particles3010007.

67. Brandt, B.B.; Cuteri, F.; Endrodi, G.; Schmalzbauer, S. Exploring the QCD phase diagram via reweighting from isospin chemical potential. POS 2019, LATTICE2019, 189, [1911.12197]. doi:10.22323/1.363.0189.

68. Cao, X.; Liu, H.; Li, D.; Ou, G. QCD Phase Diagram at Finite Isospin Chemical Potential and Temperature in an IR-improved Soft-Wall AdS/QCD Model. Chinese Phys. C 2020, 44, 083106, [2001.02888]. doi:10.1088/1674-1137/44/8/083106.

69. Brandt, B.B.; Cuteri, F.; Endrodi, G. QCD thermodynamics at non-zero isospin asymmetry. 38th International Symposium on Lattice Field Theory, 2021, [2110.14750].

70. Johnson, S.G. The NLopt nonlinear-optimization package, 2011.

71. Guennebaud, G.; Jacob, B.; others. Eigen v3. http:/ / eigen.tuxfamily.org, 2010.

72. The pandas development team. pandas-dev/pandas: Pandas, 2020. doi:10.5281/zenodo.3509134.

73. Harris, C.R.; Millman, K.J.; van der Walt, S.J.; Gommers, R.; Virtanen, P.; Cournapeau, D.; Wieser, E.; Taylor, J.; Berg, S.; Smith, N.J.; Kern, R.; Picus, M.; Hoyer, S.; van Kerkwijk, M.H.; Brett, M.; Haldane, A.; Fernández del Río, J.; Wiebe, M.; Peterson, P.; Gérard-Marchant, P.; Sheppard, K.; Reddy, T.; Weckesser, W.; Abbasi, H.; Gohlke, C.; Oliphant, T.E. Array programming with NumPy. Nature 2020, 585, 357-362. doi:10.1038/s41586-020-2649-2.

74. Hunter, J.D. Matplotlib: A 2D graphics environment. Computing in Science \& Engineering 2007, 9, 90-95. doi:10.1109/MCSE.2007.55. 